\title{
Displasia renal multiquística: curso clínico y evaluación del riñón contralateral
}

\author{
Iris de Castaño ${ }^{1}$, Lina María Velásquez ${ }^{2}$
}

\section{RESUMEN}

Introducción: La displasia renal multiquística (DRM) unilateral es una malformación frecuentemente encontrada en el ultrasonido obstétrico. En Colombia no existen publicaciones sobre las características clínicas y el comportamiento de esta enfermedad que permitan sugerir el tipo de estudios que se deben practicar en la fase inicial y en el seguimiento de estos niños.

Objetivos: Describir las características clínicas de 40 pacientes con DRM unilateral con énfasis en la evaluación del riñón contralateral (RC) con respecto a sus funciones, tamaño y frecuencia de malformación urológicas asociadas.

Metodología: Estudio descriptivo de pacientes menores de 18 años con diagnósticos pre o postnatales de DRM unilateral, atendidos en la consulta externa de nefrología pediátrica del Hospital Infantil Club Noel entre julio de 1997 y diciembre de 2007.

Resultados: Se encontraron 40 pacientes. En ellos $88 \%$ el diagnóstico se hizo antes de nacer; $55 \%$ eran de sexo masculino, con una edad promedio al ingreso de 2 meses (rangos, 1 y 42 meses). En el RC se descubrió hidronefrosis (HN) en 2 casos (5\%) y reflujo vesicoureteral (RVU) grados I y II en 3 casos (9\%). El cálculo de la filtración glomerular fluctuó entre $81 \mathrm{ml} / \mathrm{min} /$ 1.73 y $193 \mathrm{ml} / \mathrm{min} / 1.73$ (media, 105). Sólo uno de los 38 renogramas fue anormal e informó la presencia de una cicatriz renal. En el control ecográfico de 31 pacientes los quistes desaparecieron en 13 y persistían en 18, con una media de 60 meses (rango, 24 y 132 meses). El tiempo de seguimiento promedio fue 60 meses con un rango entre 15 y 132 meses.

Conclusiones: En este grupo de pacientes se encontró buen pronóstico del RC: ninguno presentó disminución de la filtración glomerular y la frecuencia de malformaciones urológicas como la HN yel RVU fue muy baja. El ultrasonido renal(UR) es indispensable en la evaluación inicial y seguimiento de los casos. Es recomendable practicar al comienzo un renograma, preferiblemente con ${ }^{99 m} \mathrm{Tc}$-DMSA, para comprobar la no funcionalidad del riñón displástico y evaluar la morfología del RC. Se sugiere reservar la cistouretrografía miccional (CUGM) para los casos en los que se descubra HN postnatal y/o alteraciones del renograma en el RC. Es importante el cálculo periódico de la filtración glomerular para detectar hiperfiltración que puede comprometer a largo plazo el único riñón funcional.

Palabras clave: Displasia renal multiquística; Niños; Riñón contralateral; Función renal; Reflujo vesicoureteral; Cistouretrografia miccional; Renograma.

Multicystic Dysplastic Kidney: clinical course and prognosis of the contralateral kidney

\section{SUMMARY}

Introduction: The multicystic dysplastic kidney (MDK) is a frequent malformation discovered in obstetrical ultrasound. In Colombia, there are no publications on clinical characteristics and follow up studies suggesting initial evaluation and follow up in these children.

Objectives: To describe the clinical characteristics of 40 patients with unilateral MDK, with emphasis in the evaluation of the contra-lateral kidney (CK) regarding function, size, and associated urological malformations.

Methodology: A review of cases of patients under 18 years of age with prenatal or postnatal diagnosis of unilateral MDK that attended the Pediatric Nephrology outpatient clinic at Hospital Infantil Club Noel in the city of Cali from July 1997 to December 2007.

Results: A total of 40 patients were found. In $88 \%$ of these, diagnosis of unilateral MDK was done prenatally; of these, $55 \%$ were males with a mean age of 2 months of age (range 1 to 42 months). Evaluation of contra-lateral kidney showed:

1. Profesora Asociada, Departamento de Pediatría, Escuela de Medicina, Facultad de Salud, Universidad del Valle. e-mail:ircastan@yahoo.com

2. Médica pediatra, Universidad Surcolombiana, Neiva. e-mail: limavela3001@yahoo.es

Recibido para publicación noviembre 9, 2005 Aceptado para publicación septiembre 30, 2009 
hydronephrosis ( $\mathrm{HN})$ in 2 cases $(5 \%)$, vesicouretheral reflux (VUR) grades I and II in 3 cases (9\%). Glomerular filtration fluctuated between $81 \mathrm{ml} / \mathrm{min} / 1.73$ and $193 \mathrm{ml} / \mathrm{min} / 1.73$ (mean 105). Radioisotope renography was performed in 38 patients and only one patient was reported with renal scar. Follow up with renal ultrasound revealed that renal cysts disappeared in 13 patients and persisted in 18 with a media of 60 months (range 15 to 132 months).

Conclusions: In this group of patients, a good prognosis of the CK was found: none presented decline of the glomerular filtration rate and frequency of urological malformations as $\mathrm{HN}$ and VUR was very low. Renal ultrasound is mandatory in the initial evaluation and follow up of these cases. Renography with $99 \mathrm{mTc}$-DMSA is recommended in the initial evaluation of these patients to demonstrate no function of the MDK and to assess morphology and function of CK. A voiding cystogram should be reserved to patients with hydronephrosis in $\mathrm{CK}$ and/or anomalies in the renography. The periodic calculation of the glomerular filtration is important to detect hyperfiltration that can compromise a single functional kidney.

Keywords: Multicystic dysplastic kidney; Children; Contralateral kidney; Renal function; Vesicouretheral reflux; Micturating cystourethrogram; Renography.

La interacción entre la yema ureteral y el blastema metanéfrico es crucial para la formación de riñones funcionales. Una inapropiada penetración e inducción del blastema metanéfrico causa diferenciación anormal no funcionante del tejido renal y formación del riñón displástico. La DRM es unilateral en la mayoría de los casos, sin base genética a diferencia de la enfermedad poliquística que se presenta en forma bilateral y puede ser autosómica recesiva (tipo infantil) o autosómica dominante (tipo adulto). El uso del ultrasonido obstétrico ha permitido el descubrimiento cada vez mayor de anormalidades del tracto urinario fetal incluyendo la DRM unilateral. La incidencia de la enfermedad descubierta prenatalmente es de 1 por cada 3,640 recién nacidos vivos ${ }^{1}$. Pueden existir malformaciones asociadas en el RC incluyendo la HN entre 3\% y 12\% de los $\operatorname{casos}^{2}$ y en mayor grado la presencia de RVU entre $10 \%$ y $26 \%$ de los $\operatorname{casos}^{2-4}$. El examen imageneológico inicial es el UR por cuyo medio se confirma la presencia de múltiples quistes de diferentes tamaños que no comunican entre sí y reemplazan el parénquima renal del lado afectado e informa las características del RC con respecto al tamaño, ecogenicidad y presencia o no de hidronefrosis $\mathrm{u}$ otra malformación. La presencia de
RVU contralateral puede colocar potencialmente a riesgo el único riñón funcionante por el desarrollo de infección del tracto urinario (ITU) y cicatrices renales ${ }^{5}$, razón por la cual algunos centros sugieren la práctica rutinaria de la CUGM y el comienzo temprano de profilaxis $^{2,5}$.

Aunque la literatura no ha sido concluyente con respecto a las implicaciones renales a largo plazo del RVU descubierto en el $\mathrm{RC}^{3,4,6}$ nuevas publicaciones cuestionan la práctica rutinaria de esta técnica por el comportamiento benigno del RVU encontrado en el RC ya que la mayoría son de baja graduación, resuelven espontáneamente no aumentan el riesgo de ITU ni el desarrollo de cicatrices renales ${ }^{7,8}$.

A través del renograma con ácido dimercaptosuccínico (DMSA) se confirma la no funcionalidad del riñón displástico y se evalúa la morfología del RC.

Casi todos los riñones displásticos involucionan totalmente durante los primeros cinco años de vida y no se recomienda la nefrectomía de rutina. Es necesario un protocolo inicial de investigación y seguimientos regulares, aunque el riñón displástico haya sido removido, para determinar el crecimiento y la función del $\mathrm{RC}$ a través del tiempo.

La presente revisión describe la experiencia de las autoras y permite ver la presentación clínica de la DRM, la frecuencia y grado de RVU en el RC por medio de la CUGM, la presencia de HN por medio del UR y la evaluación morfológica y funcional del RC a través del renograma, la creatinina sérica la y filtración glomerular. Con base en los resultados, se sugieren recomendaciones sobre los estudios radiológicos y los exámenes de laboratorio que se han de practicar en la fase inicial y en el seguimiento de estos casos.

El objetivo de esta investigación es describir las características encontradas en los pacientes con DRM unilateral que consultaron en el período del estudio al Servicio de Nefrología del Hospital Infantil Club Noel de Cali, con sus características clínicas, paraclínicas, radiológicas y gammagráficas. Determinar la frecuencia de anormalidades urológicas asociadas con el RC, determinar la función renal y el tamaño del RC y el promedio deedad de involución total del riñón displástico.

\section{MATERIALES Y MÉTODOS}

Se realizó un estudio descriptivo tipo serie de casos 
de pacientes pediátricos con diagnóstico de DRM que consultaron o fueron remitidos al Servicio de Nefrología Pediátrica del Hospital Infantil Club Noel de Santiago de Cali en el período comprendido entre julio de 1997 y diciembre de 2007.

Criterios de inclusión. Paciente con diagnóstico de DRM unilateral que consultó al Servicio de Consulta Externa de Nefrología Pediátrica desde el período de recién nacido hasta los 18 años de edad.

Criterios de exclusión. Pacientes con DRM bilateral o aquellos con historias clínicas de información incompleta.

La recolección de datos se hizo a través de un instrumento diseñado antes que describía las características del paciente: nombre y apellidos, género, edad, tipo de diagnóstico pre o postnatal, lado afectado, anomalías urológicas presentes en el RC a través del UR y de la CUGM y hallazgos funcionales y morfológicos por medio de la gammagrafía renal. En la evolución de los casos se analizó el valor de la presión arterial, la presencia deinfección urinaria por medio del urocultivo, el valor de la creatinina sérica en $\mathrm{mg} / \mathrm{dl}$, el valor estimado de la filtración glomerular en $\mathrm{ml} / \mathrm{min} / 1.73 \mathrm{~m}^{2}$ (fórmula de Schwartz), la presencia de proteinuria en $\mathrm{mg} / \mathrm{dl}$ en el examen general de orina y la evolución ecográfica del riñón displástico y el crecimiento del RC. El cálculo de la longitud renal $(\mathrm{cm})$ en enfermos mayores de 1 año de edad se hizo con la siguiente fórmula $=6.79+0.22 \mathrm{x}$ edad (años) y en los menores de 1 año $=4.98+0.155 \mathrm{x}$ edad (meses).

En el Servicio de Nefrología Pediátrica «Prorenal» del Hospital Infantil Club Noel se seleccionaron los protocolos archivados con diagnóstico de RDM y se revisaron las respectivas historias clínicas en el archivo del hospital. Los datos los colectó un médico pediatra de la Universidad Surcolombiana de Neiva que rotó por el Servicio de Nefrología Pediátrica del Hospital Universitario del Valle y del Hospital Infantil Club Noel y por una nefróloga pediatra, médica asistencial del Hospital Infantil Club Noel y docente de pediatría de la Universidad del Valle.

Ética. De acuerdo con la Declaración de Helsinki de 1975 modificada en el año 2004 y la Resolución No 8430 de 1993 del Ministerio de Salud para el desarrollo de investigaciones en humanos, el presente es un estudio sin riesgo que se basó en la recolección de informes de las historias clínicas. Fue revisado y aprobado por el

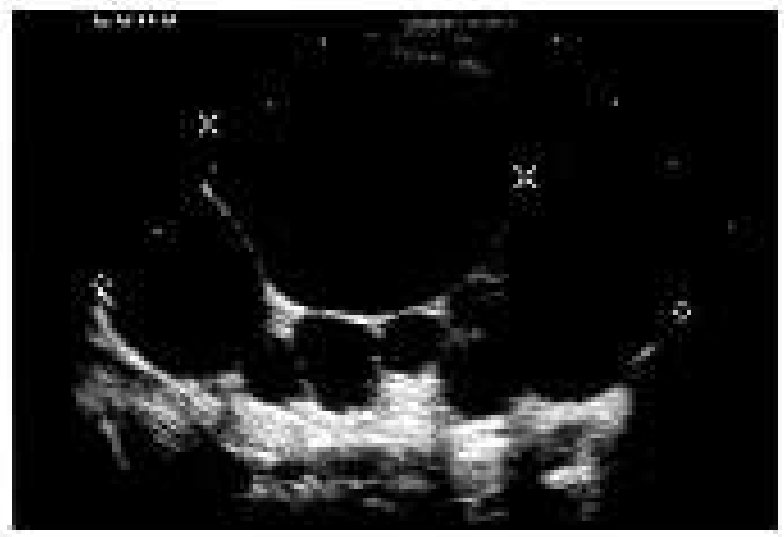

Figura 1. Ultrasonido de DRM. Presencia de múltiples quistes que no comunican entre sí y reemplazan el parénquima renal

Comité de Ética Médica del Hospital Infantil Club Noel.

Estadística. Los datos obtenidos se digitaron en una base de datos creada en el paquete estadístico EpiInfo versión 3.3 donde se agruparon las distintas fuentes de información y se verificaron los errores de digitación y de concordancia entre los registros obtenidos. Inicialmente se hizo un análisis exploratorio de los datos con los diferentes tipos de variables y luego se realizó un análisis descriptivo. Para las variables cuantitativas, se calcularon las medidas de tendencia central, posición y dispersión. Las variables cualitativas se organizaron en distribuciones de frecuencias agrupadas. Se orientó el análisis en torno a las variables que generaron respuestas para los objetivos del estudio.

\section{RESULTADOS}

Se obtuvo un total de 42 historias clínicas con diagnóstico de DRM, no se encontró ningún caso de RDM bilateral y como dos se descartaron por datos incompletos quedaron para el análisis 40 casos. Las principales características clínicas de la serie se resumen en el Cuadro 1. Se hizo diagnóstico prenatal en 35 (88\%) pacientes y en 5 niños se descubrió la enfermedad posnatalmente por medio del UR al investigar otras enfermedades ( 2 pacientes por infección del tracto urinario, 1 por masa abdominal palpable, 1 por hemangioma en piel y 1 en estudio de ictericia) (Figura 1).

No se vieron diferencias con el lado comprometido. De los pacientes 55\% eran del sexo masculino. La presión arterial fue normal en todos los 28 casos en los 


\section{Cuadro 1 \\ Características clínicas en $\mathbf{4 0}$ pacientes con DRM unilateral}

\begin{tabular}{|c|c|}
\hline Variable & $\begin{array}{c}\mathrm{N}^{\circ} \text { de } \\
\text { pacientes (\%) }\end{array}$ \\
\hline \multicolumn{2}{|l|}{ Presentación clínica } \\
\hline Diagnóstico prenatal & $35(88)$ \\
\hline Diagnóstico postnatal & $5(12)$ \\
\hline \multicolumn{2}{|l|}{ Sexo } \\
\hline Masculino & $22(55)$ \\
\hline Femenino & $18(45)$ \\
\hline \multicolumn{2}{|l|}{ Presión arterial (28 registros) } \\
\hline Normal & $28(100)$ \\
\hline \multicolumn{2}{|l|}{ Lado RDM } \\
\hline Derecho & $20(50)$ \\
\hline Izquierdo & $20(50)$ \\
\hline \multicolumn{2}{|l|}{ Hidronefrosis (40 UR) } \\
\hline No & $38(95)$ \\
\hline Sí & $2(5 \%)$ \\
\hline \multicolumn{2}{|l|}{ RVU (32 CUGM) } \\
\hline Normal & $29(91)$ \\
\hline RVU & $3(9)$ \\
\hline \multicolumn{2}{|l|}{ Presencia de ITU } \\
\hline No & $30(75)$ \\
\hline Sí & $10(25)$ \\
\hline \multicolumn{2}{|l|}{ Evolución de la DRM (31 casos) } \\
\hline Involución total & $13(42)$ \\
\hline No involución & $18(58)$ \\
\hline \multicolumn{2}{|c|}{ Tamaño del $R C$ en el seguimiento ( 30 casos) } \\
\hline Normal & $20(67)$ \\
\hline Aumentado & $10(33)$ \\
\hline \multicolumn{2}{|l|}{ Renograma (38 pacientes) } \\
\hline Normal & $37(97)$ \\
\hline Anormal & $1(3)$ \\
\hline \multicolumn{2}{|c|}{ Filtración glomerular (25 pacientes) } \\
\hline Entre $81-149\left(\mathrm{ml} / \mathrm{min} / 1.73 \mathrm{~m}^{2}\right)$ & $21(84)$ \\
\hline Entre $151-193\left(\mathrm{ml} / \mathrm{min} / 1.73 \mathrm{~m}^{2}\right)$ & $4(16)$ \\
\hline
\end{tabular}

DRM: displasia renal multiquística UR: ultrasonido renal $\mathrm{RC}$ : riñón contralateral CUGM: cistouretrografía miccional

que se encontró registro. No hubo proteinuria en el uroanálisis de los 40 enfermos.

El valor de la creatinina sérica registrada en 29 casos fue normal. El valor de la filtración glomerular en 25 pacientes fluctuó entre 81 y $193 \mathrm{ml} / \mathrm{min} / 1.73$ con una media de $105 \mathrm{ml} / \mathrm{min} / 1.73$. En cuatro pacientes $(16 \%)$ la filtración estaba por encima de $150 \mathrm{ml} / \mathrm{min} / 1.73$.

En el UR postnatal se vio HN contralateral en 2 pacientes $(5 \%)$ y fue de tipo no obstructivo en ambos casos. En la CUGM de 32 enfermos se había RVU en 3 casos ( $9 \%$ ) en uno de ellos grado I y grado II en los otros. Además 10 (25\%) presentaron ITU ( 1 con RVU y 9 sin RVU).

En 38 gammagrafías renales ( 19 con TcDMSA, 16 con Tc-MAG3 y 3 con Tc-DTPA) fue posible apreciar la no funcionalidad del riñón displástico en $100 \%$ de los casos y se comunicó morfología normal del RC en $97 \%$ de los casos (Figura 2). Sólo se informó la presencia de una cicatriz renal en 1 paciente con ITU sin RVU.

En la evolución ecográfica de 31 riñones displásticos (5 no tenían dato ecográfico de control y 4 habían sido nefrectomizados) hubo involución total de los quistes en 13 casos (42\%) entre 5 y 96 meses de edad (edad promedio de 38 meses) y quedaron sin involución 18 casos (58\%) entre 24 y 132 meses (edad promedio 60 meses); en ningún caso se informó presencia de neoplasia en el período de observación. En la evolución ecográfica del RC de 30 enfermos se encontró mayor longitud de lo establecido para la edad del paciente en 10 casos (33\%).El tiempo de seguimiento fluctuó entre 15 y 132 meses con un promedio de 60 meses.

\section{DISCUSIÓN}

Este estudio es el primer informe en Colombia acerca de la presentación clínica del RDM unilateral y el pronóstico del RC. El RDM unilateral es la enfermedad quística que se ve con más frecuencia en niños, el pediatra está obligado a orientar el manejo y enfoque de esta entidad desde el período de recién nacido y conocer su evolución a través del tiempo ${ }^{9,10}$.

El UR es un método imageneológico no 


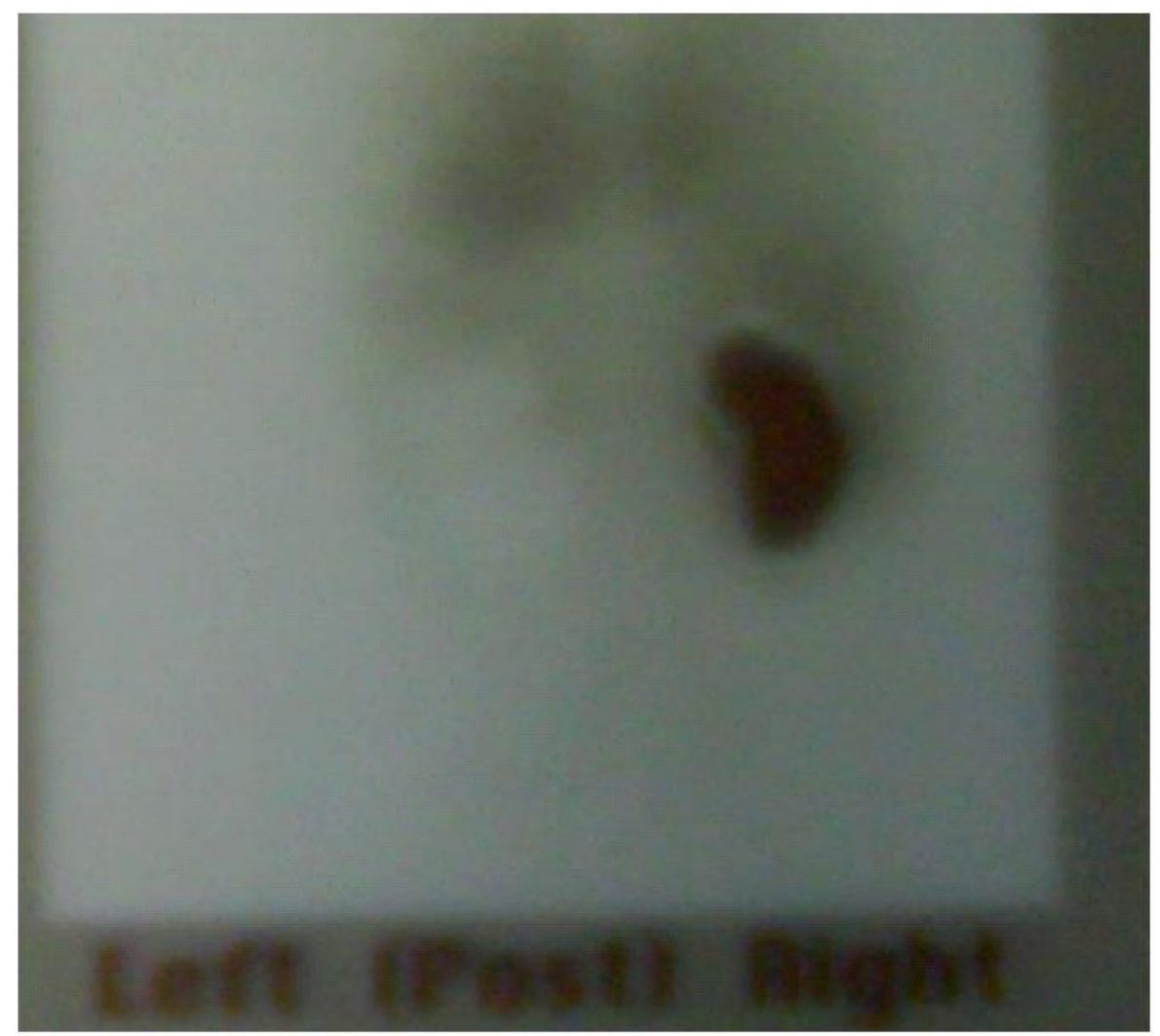

Figura 2. Gammagrafía renal Tc DMSA. Riñón izquierdo no funcionante por presencia de displasia renal multiquística; riñón derecho normal.

invasivo que se recomienda practicar minuciosamente y de manera rutinaria la primera semana de vida para confirmar si hay o no el RDM que se sospecha prenatalmente y evaluar el $\mathrm{RC}$ con respecto al tamaño, visualización o no del uréter, presencia o no de dilataciones de la pelvis y los cálices y el grado de ecogenicidad del parénquima renal; las anormalidades en estos puntos pueden hacer pensar indirectamente en la presencia de $\mathrm{RVU}^{5,11,12}$.

Ésta se confirma por medio de la CUGM; infortunadamente a través del tiempo ningún estudio radiológico menos invasivo ha reemplazado a la CUGM para confirmar el hallazgo de RVU. Los informes a largo plazo han confirmado el buen pronóstico del RC y cuestionan la práctica rutinaria de la CUGM. La publicación más reciente al respecto es de Aslam et al. ${ }^{9}$; los autores comunican el seguimiento de un buen número de casos con DRM (202) controlados durante 10 años, y reafirman la buena evolución de la DRMy recomiendan abandonar la práctica rutinaria de la CUGM porque es un examen invasivo que provoca inconformidad y dolor en el paciente por la cateterización uretral, exposición a irradiación y riesgo para el desarrollo de infecciones urinarias.

Quizá en un futuro se pueda dar mayor importancia al concepto que si la gammagrafía renal ${ }^{99 \mathrm{~m}} \mathrm{Tc}$-DMSA es normal en los pacientes con DRM, rara vez existirá RVU o si existe será de mínima graduación y susceptible a desaparecer de forma espontánea sin poner en riesgo la función renal, concepto que es claro y definitivo en el enfoque presente de los niños con ITU ${ }^{13-15}$.

Infortunadamente, en los 38 pacientes en quienes se hizo renograma, no en todos fue con ${ }^{99 \mathrm{~m}} \mathrm{Tc}$-DMSA. En los 19 que tenían ${ }^{99 m} \mathrm{Tc}$ - DMSA renal se practicó CUGM en 12 ( 7 sin ITU y 5 con ITU) siendo normal en todos los casos; es posible que los $7 / 12$ casos (58\%) con DMSA renal normal, sin ITU y sin HN no ameritaban la CUGM.

En los pacientes con RDM se sugiere practicar por lo menos un renograma inicial, en lo posible con ${ }^{99 \mathrm{~m}} \mathrm{Tc}$ DMSA, al mes de vida, para confirmar la no funcionalidad del riñón displástico, diferenciarlo de un posible riñón 
hidronefrótico y evaluar las alteraciones morfológicas del riñón contralateral como son los defectos de concentración y/o las cicatrices renales que pudiesen indirectamente hacer sospechar la presencia de RVU y de esta manera determinar cuál paciente requiere la CUGM.

Los parámetros para catalogar el DRM como benigno, además de la involución de la lesión, son ningún deterioro de la función renal contralateral ${ }^{16,17}$, ningún desarrollo de tumor de Wilms ${ }^{17,18}$ y el bajo riesgo de HTA (5.4 por 1,000 pacientes) $)^{17,19}$, en vista de la ausencia de estos parámetros en la población del presente estudio, es posible catalogar esta entidad como benigna.

El manejo conservador en lugar de la nefrectomía es la conducta universalmente aceptada ${ }^{17,20}$. Es posible que en un futuro, con el advenimiento de un método no invasivo como la cirugía laparoscopica, se podría imponer esta modalidad terapéutica conforme hace poco lo propusieron Kaneko et al. ${ }^{21}$

En vista de que un alto porcentaje de pacientes (58\%) con una edad promedio de 60 meses puede persistir con quistes renales, se sugiere continuar los controles a largo plazo con UR cada 1 ó 2 años hasta la involución total de los quistes.

En adultos con riñónúnico funcionante (por agenesia renal contralateral) con más de 15 años de observación se describe mayor riesgo de proteinuria, HTA y deterioro de la función renal ${ }^{22}$, deterioro que se podría explicar por la hiperfiltración del único riñón funcionante, hiperfiltración que se puede iniciar en la edad pediátrica como se informó en 4 de estos pacientes. El mayor tamaño de la longitud renal para la edad del enfermo en 10 casos, también puede predisponer a hiperfiltración. La presencia de microalbuminuria es un marcador temprano de daño renal, infortunadamente, fue un examen que no se ordenó de rutina en esta población. Es necesario el control médico durante la edad adulta, con mediciones de creatinina sérica, toma de presión arterial y búsqueda de proteinuria.

\section{CONCLUSIONES}

En este grupo de pacientes, en la mayoría de los casos, la DRM se descubrió en la edad prenatal; se encontró buen pronóstico del RC, pues ninguno tuvo descenso de la filtración glomerular y la frecuencia de malformaciones urológicas como la $\mathrm{HN}$ y el RVU fue muy baja. Se recomienda escoger los estudios radiológicos menos invasivos pero que sean confiables para determinar el pronóstico del RC. El UR es indispensable en la evaluación inicial y en el seguimiento de los casos. Es recomendable practicar inicialmente un renograma, de preferencia con ${ }^{99} \mathrm{~m} \mathrm{Tc}-\mathrm{DMSA}$, para comprobar la no funcionalidad del riñón displástico y evaluar la morfología del RC. Se sugiere reservar la CUGM para los casos en los que se descubra HN postnatal y/o alteraciones gammagráficas en el RC. Aunque en la edad pediátrica la función del RC, como se describe en el presente estudio es normal, en la edad adulta puede presentarse la hiperfiltración y aparición de proteinuria e HTA que pueden comprometer a largo plazo el único riñón funcionante; de allí la sugerencia del seguimiento a largo plazo de esta población.

Conflicto de intereses. Los autores declaran que no hay conflicto de intereses en el presente manuscrito.

\section{AGRADECIMIENTOS}

Al doctor Carlos Armando Echandía, Profesor Asociado, Departamento de Pediatría, Facultad de Salud, Universidad del Valle, Magíster en Epidemiología, por su colaboración para elaborar el protocolo inicial y el cuestionario. A los miembros del archivo del Hospital Infantil Club Noel por su efectiva y diligente labor al facilitar las historias clínicas de los pacientes con RDM.

\section{REFERENCIAS}

1. James CA, Watson AR, Twining P, Rance CH. Antenatally detected urinary tract abnormalities: changing incidence and management. Eur J Pediatr. 1998; 157: 508-11.

2. Atiyeh B, Husmann D, Baum M. Contralateral renal abnormalities in multicystic-dysplastic disease. J Pediatr. 1992; 121: $65-7$.

3. John U, Rudnik-Schoneborn S, Zerres K, Misselwitz J. Kidney growth and renal function in unilateral multicystic dysplastic kidney disease. Pediatr Nephrol. 1998; 12: 567-71.

4. Feldenberg LR, Siegel NJ. Clinical course and outcome for children with multicystic dysplastic kidneys. Pediatr Nephrol. 2000; 14: 1098-101.

5. Flack CE, Bellinger MF. The multicystic dysplastic kidney and contralateral vesicoureteral reflux: protection of the solitary kidney. J Urol. 1993; 150: 1873-4.

6. Abidari JM, Park KH, Kennedy WA, Shortliffe LD. Serial follow-up of the contralateral renal size in children with multicystic dysplastic kidney. J Urol. 2002; 168: 1821-5.

7. Miller DC, Rumohr RL, Dunn RL, Bloom DA, Park JM. What 


\section{Colombia Médica}

is the fate of the refluxing contralateral kidney in children with multicystic dysplastic kidney? J Urol. 2004; 172: 1630-4.

8. Ismaili K, Avni FE, Alexander M, Schulman C, Collier F, Hall M. Routine voiding cystourethrography is of no value in neonates with unilateral multicystic dysplastic kidney. JPediatr. 2005; 146: 759-63.

9. Aslam M, Watson AR, Trent \& Anglia MCDK Study Group. Unilateral multicystic dysplastic kidney: long term outcomes. Arch Dis Child. 2006; 91: 820-3.

10. Glassberg KI. Renal dysgenesis and cystic disease of the kidney. In: Wein AJ, Kavoussi LR, Novick AC, Partin AW, Peters CA (eds). Campbell-Walsh Urology. ${ }^{\text {th }}$. Philadelphia: WB Saunders; 2007. p. 3334-9.

11. De Bruyn R, Gordon I. Postnatal investigation of fetal renal disease. Prenat Diagn. 2001; 21: 984-91.

12. Kuwertz-Broeking E, Brinkmann OA, Von Lengerke HJ, Sciuk $\mathrm{J}$, Fruend S, Bulla $\mathrm{M}$, et al. Unilateral multicystic dysplastic kidney: experience in children. BJU Int. 2004; 93: 388-92.

13. Tseng MH, Lin WJ, Lo WT, Wang SR, Chu ML, Wang CH. Does a normal DMSA obviate the performance of voiding cystourethrography in evaluation of young children after their first urinary tract infection? J Pediatr. 2007; 150: 96-9.

14. Preda J, Jodal U, Sixt R, Stokland E, Hansson S. Normal dimercaptosuccinic acid scintigraphy makes voiding cystoure-

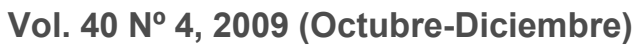

thrography unnecessary after urinary tract infection. JPediatr. 2007; 151: 581-4.

15. Hansson S, Dhamey M, Sigstrom O, Sixt R, Stokland E, Wennerstrom M. et al. Dimercaptosuccinic acid scintigraphy instead of voiding cystourethrography for infants with urinary tract infection. J Urol. 2004; 172: 1071-4.

16. Orejas G, Málaga S, Santos F, Rey C, López MV, Merten A. Multicystic dysplastic kidney: absence of complications in patients treated conservatively. Child Nephrol Urol. 1992; 12: 35-9.

17. Wacksman J, Phipps L. Report of the multicystic kidney registry: preliminary findings. J Urol. 1993; 150: 1870-2.

18. Narchi H. Risk ofWilms' tumour with multicystic kidney disease: a systematic review. Arch Dis Child. 2005; 90: 147-9.

19. Narchi H. Risk of hypertension with multicystic kidney disease: a systematic review. Arch Dis Child. 2005; 90: 921-4.

20. Al-Ghwery. Multicystic dysplastic kidney: Conservative management and follow-up. Ren Fail. 2005; 27: 189-92.

21. Kaneko K, Yamashiro Y, Yamataka A, Miyano T. Nephrectomy for multicystic dysplastic kidneys: a new therapeutic option. Pediatr Nephrol. 2005; 20: 690-1.

22. Argueso LR, Ritchey ML, BoyleETJr, Milliner DS, Bergstralh EJ, Kramer SA. Prognosis of patients with unilateral renal agenesis. Pediatr Nephrol. 1992; 6: 412-6. 